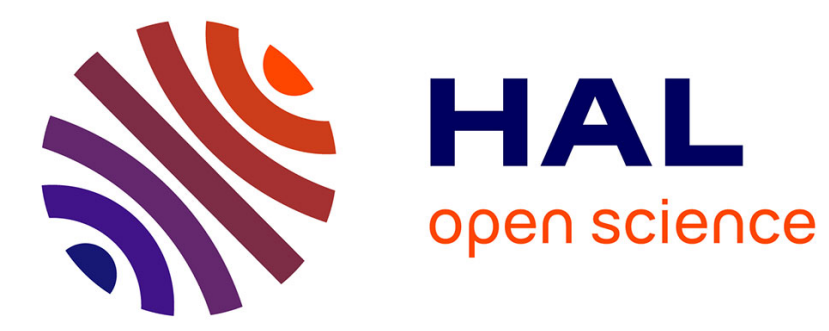

\title{
New Potential Model for Molecular Dynamic Simulation of liquid HF. II -Parameter Optimization for Repulsion-Dispersion potential
}

\author{
Vincent Dubois, Emeric Bourasseau, Jean-Bernard Maillet
}

\section{- To cite this version:}

Vincent Dubois, Emeric Bourasseau, Jean-Bernard Maillet. New Potential Model for Molecular Dynamic Simulation of liquid HF. II -Parameter Optimization for Repulsion-Dispersion potential. Molecular Physics, 2007, 105 (01), pp.125-135. 10.1080/00268970601148258 . hal-00513070

\author{
HAL Id: hal-00513070 \\ https://hal.science/hal-00513070
}

Submitted on 1 Sep 2010

HAL is a multi-disciplinary open access archive for the deposit and dissemination of scientific research documents, whether they are published or not. The documents may come from teaching and research institutions in France or abroad, or from public or private research centers.
L'archive ouverte pluridisciplinaire HAL, est destinée au dépôt et à la diffusion de documents scientifiques de niveau recherche, publiés ou non, émanant des établissements d'enseignement et de recherche français ou étrangers, des laboratoires publics ou privés. 


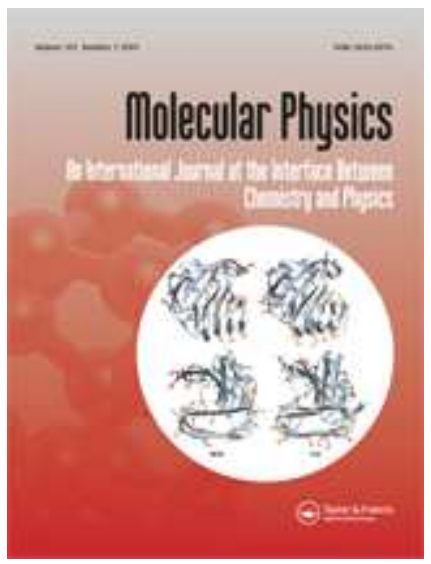

New Potential Model for Molecular Dynamic Simulation of liquid HF.

II -Parameter Optimization for Repulsion-Dispersion potential

\begin{tabular}{|c|c|}
\hline Journal: & Molecular Physics \\
\hline Manuscript ID: & TMPH-2006-0013.R1 \\
\hline Manuscript Type: & Full Paper \\
\hline $\begin{array}{l}\text { Date Submitted by the } \\
\text { Author: }\end{array}$ & 27-Nov-2006 \\
\hline Complete List of Authors: & $\begin{array}{l}\text { Dubois, Vincent; CEA } \\
\text { Bourasseau, Emeric; CEA } \\
\text { Maillet, Jean-Bernard; CEA }\end{array}$ \\
\hline Keywords: & $\begin{array}{l}\text { Parameter optimization, hydrogen fluorine, molecular dynamic } \\
\text { simulation, repulsion-dispersion contribution }\end{array}$ \\
\hline
\end{tabular}

\section{s ScholarONE \\ Manuscript Central}




\title{
New Potential Model for Molecular Dynamic Simulation of liquid HF. II - Parameter Optimization for Repulsion-Dispersion potential.
}

\author{
Vincent Dubois*, Emeric Bourasseau, Jean-Bernard Maillet \\ CEA-DAM, Département de Physique Théorique et Appliquée, \\ BP12, 91680 Bruyères-le-Châtel, France
}

\begin{abstract}
In order to build a complete potential model to perform classical molecular dynamic simulations of liquid HF, a new optimization method is proposed to obtain transferable parameters for repulsion-dispersion potential on the basis of ab initio reference data. This process is decomposed into two steps. The first step, using the force-matching method, consists in exploring the parameter space and selecting a first potential used as a starting point for the second step. This last step consists in optimizing the parameters of the selected potential in order to reproduce reference thermodynamic and structural data. The obtained potential correctly reproduces radial distribution functions and the pressures of liquid HF over a large range of thermodynamic states.
\end{abstract}

Keywords Parameter optimization, hydrogen fluorine, molecular dynamic simulation, repulsiondispersion contribution.

\section{INTRODUCTION}

Molecular methods are nowadays routinely used to predict thermodynamic and structural properties of many different systems, including metallic and organic compounds. This approach can be divided into two main categories: ab initio (or quantum) simulations and classical methods. The main difference between them is the explicit treatment of the underlying electronic structure. Indeed, quantum simulations require no a priori knowledge of atomic interactions, and appear to be accurate in predicting structural and electronic properties, but is also time consuming. Despite its wide applications, this approach still remains limited by its time and size capabilities (hundreds atoms on a picosecond timescale). This limitation could be overcome by performing classical simulations which employ analytical potentials to model interatomic forces. These potentials should be

*corresponding author: vincent-jp.dubois@cea.fr
}

simple enough to limit computing time and allows longer and larger simulations, as accurate as possible (in comparison with ab initio results), and transferable to several thermodynamic conditions (pressure and temperature). This last point is important for industrial applications where predictive capabilities of such methods are well appreciated. Unfortunately, many general force fields available in the literature are not transferable enough for direct use in industrial research. Consequently, lots of efforts have been recently devoted to construct transferable models. For example, Ungerer et al. have developed a complete and general optimization method that ensures force field transferability for entire families of organic compounds [1-7].Those potentials are now used to calibrate processes in petroleum industry. In this work, our aim is to develop and applied this type of optimization method to more complex systems like associated liquids. In particular, the hydrogen-bonded liquids play an important role in many physical, chemical and biological applications. Liquid hydrogen fluorine, 
a prototype system showing the strongest hydrogen bonds, appears to be a good candidate for a model system. Moreover, very few experimental data are available for liquid HF due to its toxicity and simulations could be useful to determine its structural and thermodynamic properties. Many classical simulations have been carried out so far with several effective potentials [8-13]. These simulations have shown that it is difficult to reproduce hydrogen bonds in this liquid over a large range of thermodynamic states. Indeed, structural properties of the liquid strongly depend on the chosen interatomic potential. To overcome this limitation, ab initio molecular dynamics simulations have been performed by Röthlisberger and Parrinello [14] and have shown the existence of one dimensional networks made of $\mathrm{HF}$ molecules sharing hydrogen bonds. However, all these classical or ab initio simulations have been performed near standard conditions. Recently, Kreitmeir et al. extended the ab initio investigation to several thermodynamics states, including supercritical states [15]. The behavior of HF liquid under pressure has been recently investigated by Maillet et al. using $a b$ initio simulations [16], and this study reveals interesting characteristics of pressure and temperature influences on structural properties.

Nevertheless, the limited simulation time (2ps) and the small size of the system (32 molecules) do not allow to predict all thermodynamic (like derivative quantities) and dynamic (like diffusion coefficient) properties with a sufficient accuracy. At this stage, an accurate classical force field would appear as an interesting alternative to predict such properties. We then propose to develop a complete and general method to construct realistic and transferable models for organic compounds, and apply it to liquid HF. The optimization of such potential is performed on the basis of pre-existing ab initio reference data points.

Starting from the total interatomic potential, a classical force field is generally expressed as a sum of several independent contributions taking their origins in physical sources. In our case, the total energy can be split into intramolecular, intermolecular, and electrostatic contributions. Details about the optimization of electrostatic potential are given in reference [17]. Here, we only briefly review the main characteristics of this model. To model electrostatic interactions, we have chosen the charge equilibrium method [18, 19] which has successfully been applied to hydrogen-bonded liquids, such as water [20]. Indeed, this model can reproduce the variation of charges with respect to local molecular environments, and provides a substantial improvement compared to the fixed charge model, especially in the case of polarisable molecule, such as HF. This method is based on the principle of electronegativity equalization of atoms within a molecule [21] and on the fact that the electronegativity of atoms depends on their local environment [22]. In the case of $\mathrm{HF}$, three parameters are needed to calculate the electronegativity of $\mathrm{H}$ and $\mathrm{F}$ : the electronegativity difference between $\mathrm{H}$ and $\mathrm{F}\left(\Delta \chi_{H F}^{0}\right)$ and the Slater exponent $\left(\zeta_{H}, \zeta_{F}\right)$ for $\mathrm{H}$ and $\mathrm{F}$ atoms. The optimization method has allowed to obtain parameters able to reproduce at the same time the variations of the $a b i n i$ tio electrostatic potential in a condensed phase of liquid HF for different thermodynamic conditions and the dipole of the isolated molecule $(1.8 \mathrm{D})$. These parameters seem to be accurate and transferable over different thermodynamic conditions of liquid and different phases (liquid and gas). The selected set of parameters used in the following part of the paper is: $\Delta \chi_{H F}^{0}=$ $5.182 \mathrm{~J}, \zeta_{H}=1.916 \AA^{-1}$ and $\zeta_{F} 4.277=\AA^{-1}$. In condensed phase, the simulation performed in this work have shown that the average dipole moment increases with density. For example at $500 \mathrm{~K}$, we obtain a dipole moment of $2.5 \mathrm{D}$ for a density of 1.300 g.cm ${ }^{-1}$ and $2.8 \mathrm{D}$ for 2.567 g.cm ${ }^{-1}$.

The second step of the optimization process, exposed in this paper, consists in choosing and fitting the contribution of repulsion and dispersion interactions. A general method to obtain realistic parameters is developed and applied to HF. This method is based on the minimiza- 
tion of an error function defined as the difference between a calculated property (for example the force on each atom) and the reference value of the same quantity. Following the ideas developed in the first step, three points appear essential to construct a reliable potential: the choice of the analytical expression of the potential, the reference data and the minimization routine. The first point consists in choosing the most adequate potential form to describe non-coulombic interactions. The analytical description of this part is well documented in the literature, and the bibliography proposes two main potential forms: Lennard-Jones (LJ) and Buckingham (EXP-6). The EXP-6 potential appears more flexible because it contains one more parameter. It also uses a term proportional to $r^{-6}$ to model the dispersion but an exponential term is used for the repulsion instead of a $r^{-12}$ term in LJ potentials. The differences between these two analytical expressions, apart from the number of parameters, are evidenced as pressure is loaded in the simulated material, making the repulsive part of the interaction predominant. Indeed, the EXP-6 potential, softer than the Lennard-Jones one, is known to model more accurately properties of materials under high pressure. As a consequence, we choose the EXP-6 potential to describe the repulsiondispersion contribution in our model.

An additional relevant question appears when dealing with molecules: where should be the force centers ? On each atom ("all atoms" potentials) or on groups of atoms ("united atoms" potentials) ? In the case of HF, many potentials use only one atom-atom interaction between fluor atoms (F-F) [10-13] ; nevertheless, we have tried to include all others interatomic interactions to improve the quality of the potential.

The last two steps of the optimization process are intimately linked. Indeed, the choice of reference data should be made concomitantly to the choice of the optimization method. In fact, all contributions to the potential (i.e. vibrational, electrostatic and repulsion-dispersion) are optimized sequentially, their optimization being ideally independent to each other. Each contributions is optimized on a specific reference database (specific means that the data depend only on the contribution). A natural order for the sequence of optimization then emerges from previous recommendations: first the vibrational part (or more generally intramolecular contributions) is optimized, then the electrostatic part and finally the repulsiondispersion contributions are optimized. Unfortunately, to our knowledge, no specific data is available concerning the repulsion-dispersion part and global data (i.e. average data) should be used for this part of the optimization. This implies that EXP-6 potential should not only describe the repulsion-dispersion energy but should also correct the imperfections of other contributions (vibrational and electrostatic). This becomes an important point in the case of $\mathrm{HF}$, for which the electrostatic model (variable charges) does not reproduce accurately the complex balancing between dipolar and quadrupolar interactions together with the high polarisability.

Finally, the optimization of the repulsiondispersion contribution is performed carefully in two steps. The first step consists in using the force-matching method [23], where the potential parameters are fitted to reproduce quantum forces on each atom. The aim of this method is to match as closely as possible first principle forces with those calculated by the classical potential. This method has successfully been applied to many systems [24-27] but needs complex potentials in the case of molecular systems, such as liquid water [27]. Nevertheless, the evaluation of the error function is not time consuming. This allows a proper exploration of the parameter space. This step is then useful for the selection of a suited potential form as well as for a coarse grain exploration of the error function, so "interesting zones" are localized. The next step consists in optimizing the parameters in order to reproduce reference thermodynamic and structural data. We used the original method proposed by Ungerer et al. [1], based on the minimization of an error function and modified by 
Bourasseau et al. [4] to reduce the computational cost. However, the calculation time of thermodynamic and structural data by molecular simulations remains long and limits a proper exploration of the parameter space. The initial choice of parameter, given by the previous "force-matching" optimization, is then determinant.

This paper is organized as follow. In section II, technical details about the potential model are given, together with a brief description of the methods used in our optimization process (the force-matching method and the optimization method). In this section, error functions are presented, with the appropriate choice of selected reference ab initio data. The section III is devoted to the results of force-matching method and the selection of one potential form. This section underlines the difficulties to find a potential with realistic and transferable parameters. The section IV focuses on the last optimization of this potential to reproduce thermodynamic (isotherm) and structural data (radial distribution functions). This section presents the final optimized parameters and the thermodynamic and structural properties obtained with this potential. Conclusions are drawn in section $\mathrm{V}$.

\section{METHODS}

\section{A. Potential Model and Molecular Dynamic}

We used the usual decomposition of the interaction energy into intramolecular and intermolecular parts. The intramolecular part is simply modelled by a harmonic potential:

$$
V^{\text {intra }}(R)=k_{e}\left(R-R_{e}\right)^{2}
$$

where $R_{e}$ is the equilibrium H-F distance and $k_{e}$ the force constant related to the vibrational frequency $\nu_{e}=\left(k_{e} / m\right)^{1 / 2}$. We extracted the value of these parameters from our ab initio MD simulations of liquid HF: $R_{e}=0.95 \AA$ is the average distance $\mathrm{HF}$ in the liquid and $\nu_{e}=3300 \mathrm{~cm}^{-1}$ is the central position of the intramolecular band of vibrational spectrum.

The intermolecular part is decomposed into two parts: Coulombic and non-Coulombic interactions. The Coulombic interactions take into account the electrostatic and polarization energies through the charge equilibrium method. These interactions are represented by two point variable charges located on $\mathrm{H}$ and $\mathrm{F}$ atoms. Details of this model are given in reference [17] and the parameters are presented in section I. To reduce the computational cost, the value of the charges evolved selfconsistently during the atomic motion by an effective Lagrangian dynamic and were calculated exactly by the charge equilibrium method every 1000 MD steps.

The remaining non-Coulombic part is represented by EXP- 6 potential:

$$
V_{i j}^{E X P-6}=\lambda_{i j} e^{-\left(\alpha_{i j} R_{i j}\right)}-\frac{C_{6, i j}}{R_{i j}^{6}}
$$

with three parameters $C_{6, i j}, \lambda_{i j}$ and $\alpha_{i j}$ or each couple $(\mathrm{i}, \mathrm{j})$ of atomic species (three in our case: $\mathrm{H}-\mathrm{H}, \mathrm{H}-\mathrm{F}, \mathrm{F}-\mathrm{F})$.

All the simulations in this work were performed in the NVT ensemble using a system containing $N=5832 \mathrm{HF}$ molecules. We used a periodically repeated cubic cell and a Nosé-Hoover thermostat to control the temperature. The equation of motion were integrated using the velocity Verlet algorithm, with a time step of 0.1 fs. We decided to use a cutoff of $10 \AA$ for Coulombic and non-Coulombic interaction calculations, with a Taper function to make the Coulombic interactions reach 0 at $10 \AA$.

\section{B. Force-matching method}

In this scheme, classical forces, which depend on potential parameters, are optimized by matching $a b$ initio forces on a set of different configurations obtained by MD $a b i n i-$ tio simulations. In order to increase the transferability of parameters we aim at obtaining, configurations are taken along ab initio trajectories calculated at different densities. We 
chose the same configurations which were already used to optimize the parameters of electrostatic part. These configurations have been taken from MD ab initio simulations produced by the VASP code [28] at $500 \mathrm{~K}$. Details of these simulations are given in reference [16]. Four configurations of 64 atoms have been taken, corresponding to four densities: $2.567 \mathrm{~g} . \mathrm{cm}^{-1}$, 2.193 g.cm ${ }^{-1}, 1.861 \mathrm{~g} . \mathrm{cm}^{-1}, 1.300$ g.cm ${ }^{-1}$. On the basis of atomic forces of these four configurations $\left(N_{c}=4\right)$, we define the following error function:

$$
F_{\text {force }}^{e r r}=\frac{100}{2 N_{c}} \sum_{n=1}^{n=N_{c}}\left[F_{F, n}^{e r r}+F_{H, n}^{e r r}\right]
$$

where $F_{H, n}^{e r r}, F_{F, n}^{e r r}$ are the relative root mean square (RRMS) deviations for the forces acting on, respectively, $\mathrm{H}$ atoms and $\mathrm{F}$ atoms of the $n^{t h}$ configuration. The error function is then an average RRMS deviation and the individual RRMS deviations are calculated by:

$$
F_{A, n}^{e r r}=\sqrt{\frac{\sum_{i=1}^{i=N_{A}}\left|\vec{F}_{A, n, i}^{c l a s s}-\vec{F}_{A, n, i}^{a b}\right|^{2}}{\sum_{i=1}^{i=N_{A}}\left|\vec{F}_{A, n, i}^{a b}\right|^{2}}}
$$

where $\vec{F}_{A, n, i}^{a b}, \vec{F}_{A, n, i}^{c l a s s}$ are, respectively, the reference $a b$ initio force and the force calculated using the classical potential which act on the $i^{\text {th }}$ atoms of specie $A$ in the $n^{\text {th }}$ configuration. $A$ is the atomic type ( $\mathrm{H}$ or F), $N_{A}$ the number of atoms of type $A: N_{A}=32$ for $\mathrm{H}$ and $\mathrm{F}$. The optimization of potential parameters is achieved by minimizing the error function (Eq. 3). Nevertheless, the surface produced by the error function in the parameter space is complex and we also developed a minimization routine to explore this surface and to locate the different minima. This minimization routine, which has already been used to obtain the electrostatic potential parameters [17], is based on the Powell method. For each parameter to optimize, it proceeds by surrounding a minimum, and then by minimizing the function using parabolic interpolations. Then, it determines directions through which minimization appears efficient, and repeats this procedure in those directions.
The routine stops the minimization when a satisfactory convergence is reached. To explore the parameter space, this local minimization can be repeated successively with several initial parameters. In the case of force-matching, a minimization is very fast and we have performed 50 successive minimizations from random initial parameters for each tested potential.

\section{Direct optimization on structural and thermodynamic data}

The direct optimization is also based on the basic idea of minimizing an error function. The first step is to define this function as the mean square relative deviation between reference and model results:

$$
F_{\text {direct }}^{\text {err }}=\sum_{n=1}^{n=N_{d}} X_{n}\left(\left\langle A_{n}\right\rangle-A_{n}^{\text {ref }}\right)^{2}
$$

where $N_{d}$ is the number of reference data points, $A_{n}^{\text {ref }}$ the reference data and $\left\langle A_{n}\right\rangle$ the corresponding property averaged during the classical simulations produced by the analytical potential which depends on $K$ parameters $\left\{y_{k}, k=1, \ldots K\right\}$. We have included two types of data: the radial distribution functions (structural data) and the pressures along isotherms (thermodynamic data). The reference data have been obtained by MD ab initio simulations with the VASP code. We have used the same thermodynamic states at $500 \mathrm{~K}$ as used on the force matching step and we have added three thermodynamic states at $973 \mathrm{~K}$ corresponding to three densities: 2.567 g.cm ${ }^{-1}, 2.193$ g.cm ${ }^{-1}$ and $1.861 \mathrm{~g} . \mathrm{cm}^{-1}$. So, the error function is a sum over these seven states $\left(N_{e}=7\right)$ :

$$
\begin{aligned}
F_{\text {direct }}^{\text {err }}=\frac{100}{N_{e}}\left(\sum_{n e=1}^{n e=N_{e}}(1-X p) F_{R D F}^{e r r, n e}\right. \\
\left.+X p \cdot F_{P}^{e r r, n e}\right)^{\frac{1}{2}}
\end{aligned}
$$

$F_{R D F}^{e r r, n e}$ is the relative root mean square deviation associated to the radial distributions functions: 
$F_{R D F}^{e r r, n e}=\frac{1}{3} \sum_{i j} \frac{\sum_{r}\left(\left\langle g_{i j}(r)\right\rangle_{n e}-g_{i j / n e}^{a b}(r)\right)^{2}}{\sum_{r}\left(g_{i j / n e}^{a b}(r)\right)^{2}}$

where $\left\langle g_{i j}(r)\right\rangle_{n e}$ and $g_{i j / n e}^{a b}(r)$ are the radial distributions functions for the $i j$ species couple obtained, respectively, by $a b$ initio and classical simulations of the $n e^{t h}$ thermodynamic state. The $r$ summation runs over interatomic distances between $1.1 \AA$ and $5 \AA . F_{P}^{e r r, n e}$ corresponds to the mean square relative deviation between pressure averaged over ab initio $\left(P_{n e}^{a b}\right)$ and classical $\langle P\rangle_{n e}$ simulations:

$$
F_{P}^{e r r, n e}=\frac{\left(\langle P\rangle_{n e}-P_{n e}^{a b}\right)^{2}}{\left(P^{n o r m}\right)^{2}}
$$

The classical pressure is calculated by the virial formula. We have chosen to normalize $F_{P}^{e r r, n e}$ with $P^{n o r m}=25 \mathrm{kbar}$ instead of the standard definition of RRMS to obtain the same weight for each configuration.

The $X p$ factor in equation (6) allows to modify the relative weight of the pressure contribution to the total error function. In the case of our optimizations, we have chosen $X p=0.1$ to obtain the same weight of $F_{R D F}^{e r r, n e}$ and $F_{P}^{e r r, n e}$ in the error function.

The main difficulty in minimizing the error function given by equation (5) is due to the CPU cost of its evaluation by MD simulations. So the optimization routine used in the previous force-matching step which involves at least hundreds or thousands evaluations of the error function is not well suited in this case. Instead, we chose the method proposed by Ungerer et al. [1] and based on the Gauss-Legendre least square estimator [29]. This method leads to a local minimum $\left\{y_{k}^{1}\right\}$ where partial derivatives of $F_{\text {direct }}^{\text {err }}$ with respect to potential parameters are equal to zero:

$$
\begin{aligned}
\left(\frac{\partial F}{\partial y_{k}}\right)_{1} & =0 \\
& =\sum_{n=1}^{n=N} 2 X_{n}\left(\left\langle A_{n}\right\rangle_{1}-A_{n}^{r e f}\right)\left(\frac{\partial\left\langle A_{n}\right\rangle}{\partial y_{k}}\right)_{1}
\end{aligned}
$$

To find the $\left\{y_{k}^{1}=y_{k}^{0}+\Delta y_{k}\right\}$ parameters from the initial $\left\{y_{k}^{0}\right\}$ parameters, we assume that the $\left\langle A_{n}\right\rangle_{1}$ can be expressed using the first-order Taylor expansion from the initial data:

$$
\left\langle A_{n}\right\rangle_{1}=\left\langle A_{n}\right\rangle_{0}+\sum_{k=1}^{k=K} \Delta y_{k} *\left(\frac{\partial\left\langle A_{n}\right\rangle}{\partial y_{k}}\right)_{0}
$$

The minimum condition (Eq. 9) can also be expressed as a linear system:

$$
A \Delta y=B
$$

with $K$ unknowns $\left\{\Delta y_{k}, k=1, \ldots K\right\}$ and the matrix $A$ and $B$ given by:

$$
\begin{gathered}
B_{k}=\sum_{n=1}^{n=N}\left(A_{n}^{r e f}-\left\langle A_{n}\right\rangle_{0}\right)\left(\frac{\partial\left\langle A_{n}\right\rangle}{\partial y_{k}}\right)_{0} \\
A_{k l}=\sum_{n=1}^{n=N}\left(\frac{\partial\left\langle A_{n}\right\rangle}{\partial y_{k}}\right)_{0}\left(\frac{\partial\left\langle A_{n}\right\rangle}{\partial y_{l}}\right)_{0}
\end{gathered}
$$

with $k$ and $l$ running over the number of parameters. The equation (11) can be easily solved by standard mathematical algorithms. Nevertheless, the difficulty is the evaluation of partial derivatives $\partial\left\langle A_{n}\right\rangle / \partial y_{k}$. Previous works $[1,3]$ have shown that the usual finite differences method introduces a large statistical inaccuracy and is time consuming, with $K+1$ simulations needed for each thermodynamic state. We thus have preferred the method introduced by Bourasseau et al. [4] where the derivative are calculated with statistical fluctuations formula.

Simulations with initial potential parameters $\left\{y_{k}^{0}\right\}$ allow to evaluate structural and thermodynamic data and corresponding partial derivatives. Next, the resolution of Eq. (11) provides an evaluation of the optimum set of potential parameters $\left\{y_{k}^{1}\right\}$ which minimizes the 
error function. Through Eq. (10), we evaluate the predicted data and the predicted error function (Eq. 5). Because of the approximation of the first-order Taylor expansion, the process of optimization should be repeated to converge toward the local minimum. Nevertheless, the previous work of Bourasseau et al. [4] has shown two cases of convergence:

- Case (1): Some of the optimized parameters $\left\{y_{k}^{1}\right\}$ have unrealistic values and the optimization process failed. This case is found when the starting point is far from the minimum.

- Case (2): The predicted error function is low and is in good agreement with the evaluated error function by simulations with optimized parameters $\left\{y_{k}^{1}\right\}$. In this case, the optimization is successful after one step of optimization process.

These two cases emphasize the importance of the choice of the initial parameter set.

\section{FIRST STEP: RESULTS OF FORCE-MATCHING METHOD}

Before applying the force-matching method, the error function has been evaluated (Eq. 6) without any contribution of repulsiondispersion (the total energy is the sum of intramolecular and electrostatic parts). We obtained an error function of $98 \%$ which is decomposed into RRMS deviations of $81 \%$ and $116 \%$ for forces acting on $\mathrm{H}$ atoms and $\mathrm{F}$ atoms respectively. Consequently, a model with only intramolecular and electrostatic contributions is inefficient to describe accurately total forces on each atoms, and the repulsion-dispersion potential is expected to play an important role. Nevertheless, this expected large contribution of repulsion-dispersion potential is surprising because it usually participates for about $20 \%$ of the total energy. We suspect that our "repulsion-dispersion" potential should mainly be a correction to the electrostatic and intramolecular models.

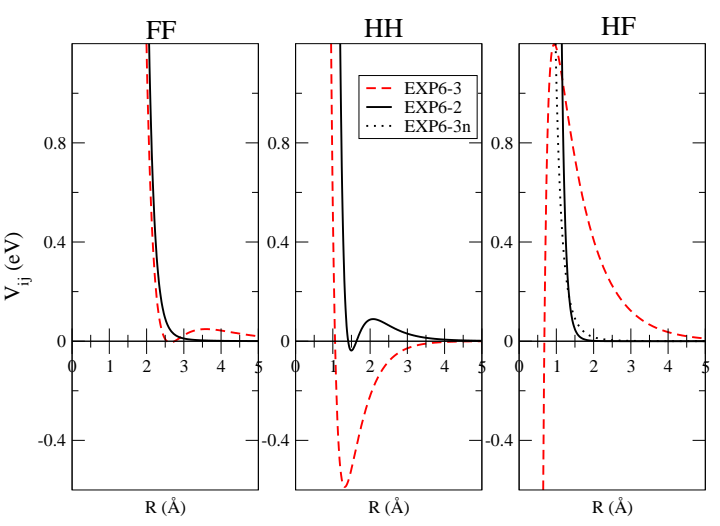

FIG. 1: Potential Values as a function of the interatomic distance. The potential parameters are the optimized values corresponding to the lowest minima obtained by force-matching of three EXP-6 components (EXP6-3) and two EXP-6 components (EXP6-2) and obtained by direct optimization of the HF component (EXP6-3n).

Preliminary tests of minimization with LJ components have shown that LJ potentials lack flexibility and fail to reproduce this complex behavior.

To avoid this lack of flexibility, we used three EXP-6 components (HH, HF and FF) in our minimization process involving 9 parameters. Hereafter this potential is referred to as EXP63. To obtain even more flexibility, no more constraint have been introduced in the optimization process excepted for $\alpha$, which value is kept between 0 and $10 \AA^{-1}$. Fifty successive minimizations were performed and the 50 minima appear to be close to each other from the point of view of the minimized value of the error function distributed around $47 \%$. This distribution is not significant and all minima can be considered as equivalent. In comparison with the case without any repulsion-dispersion potential, the three EXP-6 allow a significant decrease of error function (improvement of $51 \%$ ) with a RRMS deviation of $45 \%$ and $48 \%$ for forces acting on $\mathrm{H}$ atoms and $\mathrm{F}$ atoms, instead of $81 \%$ and $116 \%$ respectively. Nevertheless, the error remains significant and this new potential does not give an accurate reproduction of $a b$ initio forces. 
A careful analysis of the minima reveals that they are not concentrated on a particular zone in the parameter space but rather spread along a large domain. Nevertheless, the shape of the subsequent interatomic potentials remains similar. An example, corresponding to the lowest minimum, is shown in figure 1 , where the FF, $\mathrm{HF}$ and $\mathrm{HH}$ components of the potential are displayed. The FF potential is a reverse EXP-6 with $\lambda$ and $C_{6}$ largely negatives and shows repulsive energies at long distance and an energy barrier at $3.5 \AA$. The parameter values of the $\mathrm{HH}$ potential are also opposite to standard values and give a too deep attractive well: $V_{H H}=-$ $0.6 \mathrm{eV}$ at $R=1.5 \AA$. The HF potential is a repulsive soft sphere. Molecular dynamic simulations performed with these parameters show that the potential is unable to reproduce hydrogen bonds in liquid $\mathrm{HF}$, and rather reveals unrealistic H-H bonds. Therefore, the best potential obtained with a force-matching process fails to describe the structure of the liquid. To introduce more physical meaning in our potential, we have decided to model the HF contribution by a repulsive sphere with a potential given by $A_{H F} *\left(B_{H F} / R\right)^{12}$. We fixed the parameter $A_{H F}=6.2 \mathrm{eV}$ and $B_{H F}=1 \AA$ to match the hydrogen bond in the liquid. We optimized the two other EXP-6 components ( $\mathrm{HH}$ and FF) by our force-matching routine and this potential is hereafter referred to as EXP6-2.

All optimized values of the error function (Eq. 6) are very close to each other, distributed around $75 \%$. From the point of view of the description of forces, we can consider that both potentials are equivalent and allow a significant decrease of error $(24 \%)$ in comparison to the case without repulsion-dispersion potential. This decrease is mainly observed for forces acting on $\mathrm{F}$ atoms (error of $70 \%$ instead of $116 \%$ ), and almost no improvement is observed concerning hydrogen atoms ( $80 \%$ instead of $81 \%$ ). This shows that the definition of the H-F interactions is predominant for the description of forces acting on the hydrogen atom.

As in the case of EXP6-3, the optimization leads to minima scattered on a large range of parameter space, with no particular stability for any of them. Nevertheless, all minima give approximately the same potential shapes. In the following, the lowest minimum, which parameters are given in Table I, is used.

\begin{tabular}{|c|c|c|}
\hline & FF & HH \\
\hline$\lambda(\mathrm{eV})$ & 807026,46 & $-896,63$ \\
\hline$\alpha\left(\AA^{-1}\right)$ & 6,52 & 3,95 \\
\hline$C_{6}\left(\mathrm{eV} . \AA^{6}\right)$ & $-6,069$ & $-26,943$ \\
\hline
\end{tabular}

TABLE I: Optimized parameter values of EXP6-2 potential.

The optimized EXP6-2 potential exhibits a non standard behavior, with negative values for some parameters. In order to compare with EXP6-3, FF and HH components are displayed as a function of interatomic distance in Fig. 1. Concerning the FF component, both potentials give the same repulsive wall energies, but EXP6-2 gives no energy barrier at intermediate distances, contrary to the case of EXP6-3. Concerning the $\mathrm{HH}$ component (which has little effects on the error function), more differences are seen: the EXP6-2 potential exhibits an energy barrier of $0.1 \mathrm{eV}$ together with a weak attractive well, while EXP6-3 exhibits a large (unrealistic) attractive well of $0.5 \mathrm{eV}$.

In order to investigate the behavior of EXP62 potential, molecular dynamic simulations of liquid HF have been performed at $500 \mathrm{~K}$ and 2.193 g.cm ${ }^{-1}$. Results of these simulations are compared with those obtained with a reference potential (hereafter referred to as LJ-0) used in our previous work [16]. In order to characterize the structure of the liquid, radial distribution functions calculated along those simulations have been plotted in figure 2 .

The F-F radial distribution function shows the most pronounced structure. The reference potential LJ-0 fails to reproduce this function by largely overestimating the first peak. On the other hand, our optimized potential, despite a slightly enhanced broadening in the first peak, gives an overall good description of the F-F structure. The agreement between $a b$ initio and 
our potential is also good concerning the F-H radial distribution function. Indeed, the agreement is largely improved in comparison with the LJ-0 potential. The first peak corresponds to intramolecular F-H distances and the second one at about $1.5 \AA$ results from the formation of hydrogen bonds. Hence, the good agreement with $a b$ initio results indicates that hydrogen bonds are well described in the classical simulations with EXP6-2 potential. Nevertheless, we note that the classical simulations give no overlap between the intramolecular peak and the first intermolecular one, such as observed in the $a b$ initio structure. This difference is linked to the use of the charge equilibration method which needs a repulsive HF potential at these distances to avoid the divergence of atomic charges during the simulation. Classical $\mathrm{H}-\mathrm{H}$ radial distribution functions obtained with EXP6-2 exhibit a first peak at larger atomic separation than the ab initio $\mathrm{g}(\mathrm{r})$. However, a significant improvement is observed in comparison with the structure obtained using the reference potential LJ-0.

To compare the thermodynamic properties, we calculated the isotherms at $500 \mathrm{~K}$ and $973 \mathrm{~K}$
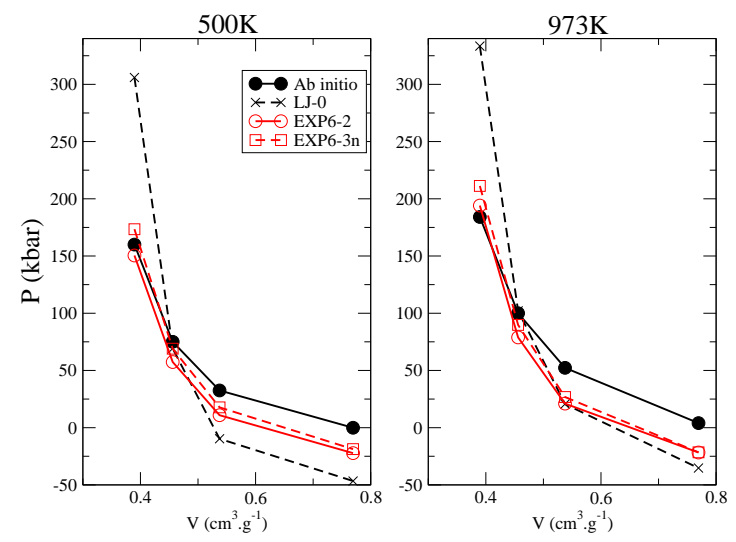

FIG. 3: Isotherms calculated at $973 \mathrm{~K}$ and $500 \mathrm{~K}$ as obtained from the $a b$ initio simulations (black) and from classical simulations with the reference LJ-0 (black dashed) potential, EXP6-2 potential (red) and EXP6-3n potential (red dashed).

by classical and ab initio simulations (Fig. 3). The reference potential (LJ-0) predicts higher pressures than quantum simulations in the high density regime, and lower pressures at low density. This disagreement may be attributed to the use of a single LJ component. With an additional component, the agreement between the isotherms predicted with EXP-2 potential and $a b$ initio simulations is improved. Nevertheless, the pressure calculated with EXP6-2 remain lower than the pressure predicted by quantum simulations for each densities.

\section{SECOND STEP : RESULTS OF DIRECT OPTIMIZATION}

We used the parameters of EXP6-2 to perform the first optimization trial according to the scheme described in section II C. The optimization process involves 6 parameters ( $\mathrm{HH}$ and $\mathrm{FF}$ components): the parameters of the $\mathrm{HF}$ repulsive sphere are kept fixed. Unfortunately, the minimization failed and did not lead to a stable set of parameters. This process corresponds to the first case referred in section II C with unrealistic values of some optimized parameters. To avoid the lack of flexility of our potential, we introduced the HF component into 
our optimization process. We match our repulsive sphere HF component by an EXP-6 potential with parameters given in Table II.

\begin{tabular}{|c|c|c|}
\hline & initial & optimized \\
\hline$\lambda(\mathrm{eV})$ & 122058 & 1506057 \\
\hline$\alpha\left(\AA^{-1}\right)$ & 10.02 & 19.02 \\
\hline$C_{6}\left(\mathrm{eV} . \AA^{6}\right)$ & 0 & -1.07 \\
\hline
\end{tabular}

TABLE II: Parameter values of HF component of EXP6-3n potential. The initial values are fitted to reproduce the repulsive sphere of EXP6-2 and the optimized values are obtained by direct optimization method. The parameter values of $\mathrm{HH}$ and FF components are identical to the parameter values used for the EXP6-2 potential (Table I).

This second optimization trial involves 9 parameters corresponding to $\mathrm{FF}, \mathrm{HH}$ and $\mathrm{HF}$ EXP-6 potentials. It converged towards a minimum with values close to those of the forcematching potential EXP6-3. This agreement between the two methods emphasizes their consistence. Unfortunately, this minimum is associated with unrealistic parameter values which reveal the failure of our potential model. In particular, the $\mathrm{HH}$ component displays a too deep attractive well which implies unrealistic $\mathrm{H}-\mathrm{H}$ bonds in liquid $\mathrm{HF}$.

To obtain a tractable potential, we have decided to reduce the number of parameters to optimize. Parameter values of EXP6-2 potential for $\mathrm{HH}$ and $\mathrm{FF}$ components are kept constant and only parameters of HF components are optimized (this new potential is referred to as EXP6-3n). The optimization process consists on performing MD simulations of the seven selected thermodynamic states with initial parameter values of EXP6-3n (see table II). We calculated the average structural and thermodynamic data and partial derivatives for several lengths of simulations. Then, we calculated the predicted optimal values of the three parameters $\left(\lambda_{H F}, \alpha_{H F}, C_{6-H F}\right)$ by resolving the system 11 for these several lengths. In Figure 4 the evolution of this predicted parameter values is shown as a function of the length of simulations.

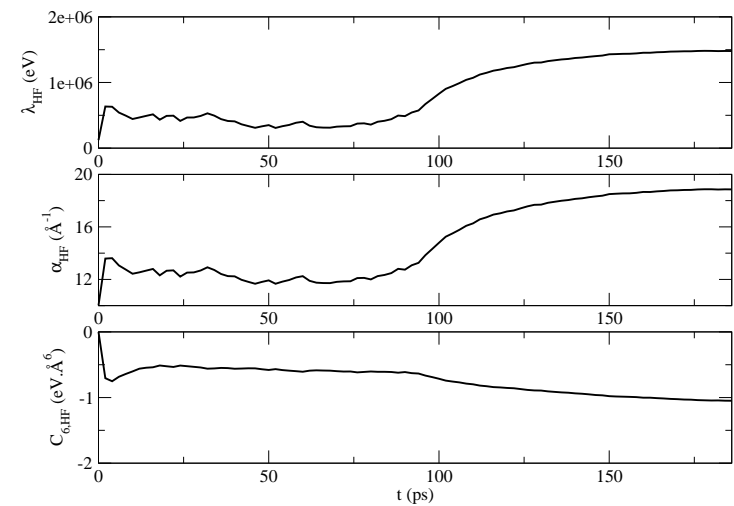

FIG. 4: Evolution of new parameter values as obtained from the direct optimization method (resolving system 11) applied to HF component of EXP6$3 n$ vs. simulation time. The point at time zero corresponds to the initial parameter values of EXP6$3 \mathrm{n}$ and simulations have been performed with these initial parameter values.

We see in Figure 4 that before $150 \mathrm{ps}$, calculated parameter values show significant oscillations due to the statistical errors of partial derivatives. This calculation converges slowly and needs MD times greater than the ones used in usual simulations: accurate values are obtained after 200 ps. Despite the speed up obtained with the new method of derivative calculations [4], the computational cost remains long. This emphasizes that the usual finite differences method should not be usable. The optimized HF component corresponds to a repulsive sphere with repulsive exponential term $(\lambda$ positive) and repulsive $1 / r^{6}$ term $\left(C_{6}\right.$ negative), as shown table II. This new component still presents physical meaning and remains close to the initial one. Nevertheless, figure 1 shows that the new potential is softer than the initial one: less repulsive at short distance and slightly more repulsive at long distance.

Molecular dynamic simulations have been performed with these new parameter values and we compared, in table III, the obtained value of the error functions (Eq. 6) with the value obtained with the initial potential and predicted though Eq. 10. The good agreement between the evaluated error by simulations with new pa- 
rameter values and the predicted errors shows that the optimization process is successful at the first step. The optimization allows a decrease of pressure error (improvement of $9 \%$ ). The more repulsive behavior of the potential at long interatomic distance also allows an increase of pressure. The agreement between the isotherms predicted with the new potential and the $a b$ initio simulations is slightly more satisfactory even if pressures remain too small at low density (Fig. 3).

The optimized potential correctly reproduces the $a b$ initio structure of liquid HF (Fig. 2) and remains close to the initial potential. In particular, the H-H and F-F structures obtained with initial and optimized potentials are almost identical. The second peak of HF RDF in Fig. 2 shows that the optimized potential allows a better description of the hydrogen bond. The origin of this improvement is the behavior of the new HF component which is softer than the initial one. We note also an enhancement of the overlap between the intramolecular peak and the first intermolecular one, in better agreement with the ab initio structure. So, the optimized EXP6-3n gives an overall good description of the structure and pressure of liquid HF.

\section{CONCLUSION}

In this work, we introduce a novel scheme for the optimization of classical potentials to described atomic interactions. Our approach is based on a sequential optimization process according to the separation of the total energy into three contributions. We follow a logical order for the optimization sequence: first the intramolecular contribution, then the electrostatic one, and finally the repulsion-dispersion part. In the case of $\mathrm{HF}$, the optimization of vibration part presents no specific difficulties. The electrostatic part is described by charge equilibrium method. The present work focusses on the optimization of repulsion-dispersion part and completes the global method to obtain the total potential. The lack of specific reference data for this part largely complicates the optimization. Indeed, the use of global data (atomic forces, pressure, RDF) implies that our repulsion-dispersion potential should not only model the repulsion-dispersion energy but also include the corrections of our intramolecular and electrostatic potential. In the case of HF, despite the use of variable charges, the electrostatic model is shown to be unable to properly reproduce the complex electrostatic behavior. The optimization of repulsion-dispersion potential should be performed very carefully and we have chosen a process in two steps where error functions are minimized based on $a b$ initio references:

- Step (1): This pre-optimization step allows the first selection of a reliable form of potential and its parameters. In order to allow a large exploration of the parameter space as well as to check for several different potentials, the evaluation of the error function should be fast. It is defined using the force-matching technique [23]. In this method, potential parameters are optimized such as to reproduce quantum forces on each atoms. It then appears that LJ potentials present a lack of flexibility and fail to repro- 
duce atomic forces on liquid HF. To overcome this limitation, a full optimization of three EXP-6 components has been performed, leading to a significant decrease of the error function. Nevertheless, this new potential (EXP6-3) has no physical meaning, especially concerning the $\mathrm{HH}$ and $\mathrm{HF}$ components, and is unable to reproduce hydrogen bonding in liquid HF. For a better description of the hydrogen bonding, we fixed a repulsive sphere for the HF component and optimized only $\mathrm{HH}$ and $\mathrm{FF}$ components. This potential (EXP6-2), associated to a greater error on atomic forces, reproduces correctly the thermodynamic (pressures) and structural (RDF) properties of HF liquid, even if the calculated pressures remain slightly lower than the $a b$ initio ones. EXP6-2 is then selected as the starting point for the second optimization step.

- Step (2): This step consists in adjusting potential parameters on the basis of thermodynamic (pressures) and structural (RDF) data of liquid. For this step, the error function is defined on the basis of these data and we minimized it with the help of an original scheme proposed in reference [1]. Despite the use of recent developments of this method to speed up the calculation [4], the computational cost remains long and prevents us from a proper exploration of the parameter space. In the case of our potential model, the full optimization of EXP62 leads to parameter values close to the ones of EXP6-3 obtained with the force-

[1] P. Ungerer, C. Beauvais, J. Delhommelle, A. Boutin, B. Rousseau, and A. H. Fuchs. J. Chem. Phys., 112:5499, 2000.

[2] E. Bourasseau, P. Ungerer, A. Boutin, and A. H. Fuchs. Mol. Sim., 28:317, 2002.

[3] E. Bourasseau, P. Ungerer, and A. Boutin. J. matching method. These values have no physical meaning and cannot be used to simulate HF liquid. To obtain a usable potential, we decided to optimize only the HF component by our direct optimization method and the parameters of the two other components ( $\mathrm{HH}$ and $\mathrm{FF}$ ) have been set to the values optimized by force-matching method. This final potential (EXP6-3n) is simple and accurately reproduce thermodynamic and structural properties of liquid $\mathrm{HF}$ over a large range of thermodynamic states.

A general method of optimization of complex potentials has been developed and applied to HF. The resulting potential is the best compromise between several properties to reproduce such as quantum forces, thermodynamic and structural properties, and dynamic tractability. As the leading part, the optimization of the repulsion-dispersion contribution did not appear straightforward, because of the former choice of intra and electrostatic contributions, and their subsequent optimizations.

For future improvements, the electrostatic model should be modified, with for example the introduction of point charges on covalent bonds. Nevertheless, we have shown that our relatively simple model can be transferable to several thermodynamic conditions. In particular it can be used at high pressures and high temperatures.

To conclude, we emphasize that our optimization method is complete and general. This method can be applied to any molecular systems, with the use of experimental as well as ab initio reference data. 
[6] M. H. Lagache, P. Ungerer, and A. Boutin. Fluid Phase Eq., 220(2):211, 2004.

[7] P. Ungerer, A. Wender, G. Demoulin, E Bourasseau, and P. Mougin. Mol. Sim., 30(10):631, 2004.

[8] W.L. Jorgensen. J. Am. Chem. Soc., 100:7824, 1978.

[9] M.L. Klein and I.R. McDonald. J. Chem. Phys., 71:298, 1979.

[10] M. E. Cournoyer and W. L. Jorgensen. Mol. Phys., 51:119, 1984.

[11] P. Jedlovszky and R. Vallauri. J. Chem. Phys., 107(23):10166, 1997.

[12] P. Jedlovszky and R. Vallauri. Mol. Phys., 93(1):15, 1998.

[13] R. G. Della Valle and D. Gazzillo. Phys. Rev. B, 59(21):13699, 1999.

[14] U. Röthlisberger and M. Parrinello. J. Chem. Phys., 106:4658, 1997.

[15] M. Kreitmer, H. Bertagnolli, J.J. Mortensen, and M. Parrinello. J. Chem. Phys., 118:3639, 2003.

[16] J.-B. Maillet, E. Bourasseau, and V. Recoules. page 224103, 2005. Phys. Rev. B.

[17] E. Bourasseau, J.-B. Maillet, L. Mondelain, and P.-M. Anglade. Mol. Sim., 31:705, 2005.
[18] W. J. Mortier, S. K. Gosh, and S. Shankar. J. Am. Chem. Soc., 108:4315, 1986.

[19] A. K. Rappé and W. A. Goddard. J. Phys. Chem., 95:3358, 1991.

[20] S. W. Rick, S. J. Stuart, and B. J. Berne. J. Chem. Phys., 101:6141, 1994.

[21] R. T. Sanderson. Science, 114:670, 1951.

[22] R. G. Parr and W. Yang. Density-Functional Theory of Atoms and Molecules. Oxford University, Oxford, 1989.

[23] F. Ercolessi and J. Adams. Europhys. Lett., 26:583, 1994.

[24] P. Tangney and S. Scandolo. J. Chem. Phys., 117:8898, 2002.

[25] J.-B. Bernard, S. Maillet. Phys. Rev. B, 66:012103, 2002.

[26] A. Aguado and P.A. Madden. Phys. Rev. B, 70:245103, 2004.

[27] S. Izvekov, M. Parrinello, C.J. Burnham, and G.A. Voth. J. Chem. Phys., 120:10896, 2004.

[28] G. Kresse and J. Furthmüller. Comput. Mat. Sci., 6:15, 1996.

[29] E. Walter and L. Pronzato. Identification of Parametric Models. Springer, Berlin, 1997. 